\title{
Scale sizes of intense auroral electric fields observed by Cluster
}

\author{
T. Johansson ${ }^{1}$, G. Marklund ${ }^{1}$, T. Karlsson ${ }^{1}$, S. Liléo ${ }^{1}$, P.-A. Lindqvist ${ }^{1}$, H. Nilsson ${ }^{2}$, and S. Buchert ${ }^{3}$ \\ ${ }^{1}$ Space and Plasma Physics, School of Electrical Engineering, Royal Institute of Technology (KTH), Stockholm, Sweden \\ ${ }^{2}$ Swedish Institute of Space Physics, Kiruna, Sweden \\ ${ }^{3}$ Swedish Institute of Space Physics, Uppsala, Sweden
}

Received: 4 June 2007 - Revised: 17 October 2007 - Accepted: 30 October 2007 - Published: 29 November 2007

\begin{abstract}
The scale sizes of intense ( $>0.15 \mathrm{~V} / \mathrm{m}$, mapped to the ionosphere), high-altitude (4-7 $R_{E}$ geocentric distance) auroral electric fields (measured by the Cluster EFW instrument) have been determined in a statistical study. Monopolar and bipolar electric fields, and converging and diverging events, are separated. The relations between the scale size, the intensity and the potential variation are investigated.

The electric field scale sizes are further compared with the scale sizes and widths of the associated field-aligned currents (FACs). The influence of, or relation between, other parameters (proton gyroradius, plasma density gradients, and geomagnetic activity), and the electric field scale sizes are considered.

The median scale sizes of these auroral electric field structures are found to be similar to the median scale sizes of the associated FACs and the density gradients (all in the range $4.2-4.9 \mathrm{~km}$ ) but not to the median proton gyroradius or the proton inertial scale length at these times and locations (22$30 \mathrm{~km}$ ). (The scales are mapped to the ionospheric altitude for reference.)

The electric field scale sizes during summer months and high geomagnetic activity $\left(K_{p}>3\right)$ are typically $2-3 \mathrm{~km}$, smaller than the typical $4-5 \mathrm{~km}$ scale sizes during winter months and low geomagnetic activity $\left(K_{p} \leq 3\right)$, indicating a dependence on ionospheric conductivity.
\end{abstract}

Keywords. Magnetospheric Physics (auroral phenomena; electric fields; magnetosphere-ionosphere interactions)

Correspondence to: T. Johansson

(tommy.johansson@ee.kth.se)

\section{Introduction}

Intense quasi-static auroral electric fields have been observed by Cluster in both the upward current region (Vaivads et al., 2003; Figueiredo et al., 2005) and the downward current region (Marklund et al., 2001; Johansson et al., 2004). The associated potential structures can be either S-shaped or Ushaped, corresponding to monopolar and bipolar electric field signatures, respectively. Both types have been observed and the $\mathrm{S}$-shaped potential structures have been shown to be associated with the sharp plasma density gradient at the plasma sheet boundary, while U-shaped potential structures occur inside the plasma sheet at less distinct gradients (Johansson et al., 2006; Marklund et al., 2007). These electric fields have a component parallel to the magnetic field, accelerating particles upward and downward. Theories attempting to explain parallel the electric fields include, e.g., strong double layers (Block, 1972), weak double layers (Temerin et al., 1982), Alfvén waves (Song and Lysak, 2001), anomalous resistivity (Hudson and Mozer, 1978) and magnetic mirror supported fields (Knight, 1973; Chiu and Schulz, 1978). Temerin and Carlson (1998) discussed the current-voltage relation in the downward FAC region and presented a model based on average charge neutrality but with the possibility of contribution to the parallel electric field from anomalous resistivity. Jasperse (1998) described downward parallel electric fields in a kinetic model, and Jasperse and Grossbard (2000) developed an analogue to the Alfvén-Fälthammar formula for the downward current region.

There are several spatial scales associated with auroral activity: fine-scale auroral arcs (order of $100 \mathrm{~m}$ ), arc systems (order of $1 \mathrm{~km}$ ) and the thickness of the auroral zone (order of $100 \mathrm{~km}$ ) (Borovsky, 1993; Galperin, 2002). In a review of 21 theories attempting to describe the formation of auroral arcs, Borovsky (1993) found that none of them could predict arc widths of some $100 \mathrm{~m}$, which was the average of the thickness of the auroral structures observed by Maggs

Published by Copernicus Publications on behalf of the European Geosciences Union. 
and Davis (1968). Several theories gave arc thicknesses of a few $\mathrm{km}$ at ionospheric altitude (e.g., strong double layers and anomalous resistivity) but some gave scale sizes one or two order of magnitudes larger than that. McFadden et al. (1999) presented a scenario (based on FAST observations of electric field structures), in which the quasi-static potential structures consist of narrow structures extending to low altitudes. However, these narrower structures were a few $\mathrm{km}$ wide, still an order of magnitude larger than the fine-scale auroral arcs.

In a statistical study of optically observed stable mesoscale auroral arcs, Knudsen et al. (2001) found a mean width of $18 \mathrm{~km}$, with a sharp cutoff at $8 \mathrm{~km}$. Together with the results of Maggs and Davis (1968), this leaves a gap in the auroral arc width distribution near $1 \mathrm{~km}$ (see Fig. 4 in Knudsen et al. (2001)). This might mean that there are different mechanisms for the fine-scale arcs and the mesoscale arcs, although uncertainties in the measurement resolution can not be ruled out as the cause of this gap.

Stenbaek-Nielsen et al. (1998) reported on optical observations showing arc widths down to $2 \mathrm{~km}$, similar to the size of auroral structures inferred from conjugate FAST electron energy flux measurements. This implies that the processes determining the arc widths at this scale occur at or above the FAST altitude of $\sim 4000 \mathrm{~km}$.

Diverging bipolar electric fields accelerate electrons upward in the downward current and they have been suggested to correspond to black aurora (Marklund et al., 1997). However, in a study using optical and radar measurements, Blixt and Kosch (2004) did not find electron density depletions for two events of black aurora, which would be expected for a downward FAC driven by a intense diverging electric field. The $1-2 \mathrm{~km}$ wide black arcs were surrounded by diffuse aurora. Peticolas et al. (2002) found only small electric fields $(<10 \mathrm{mV} / \mathrm{m})$ associated with black aurora in a study of FAST particle data (the electron signature was a $\sim 3 \mathrm{~km}$ wide energy flux depletion region) and conjugate aircraft-based optical measurements. The surrounding bright aurora was mostly diffuse. More intense electric fields associated with black aurora were found by Kimball and Hallinan (1998). Using the vorticity of four observed black curls, they could estimate the electric fields to be 80 to $300 \mathrm{mV} / \mathrm{m}$. These black curls were found together with white curls. It is possible that the intense diverging electric fields associated with the downward current region only occur for black auroras found together with discrete bright arcs (intense upward current), while there is no need for such intense electric fields for black aurora residing within diffuse white aurora.

In a statistical study of black aurora, Trondsen and Cogger (1997) found black aurora objects (black patches and arc segments) with scale sizes of $0.5-1.5 \mathrm{~km}$. Black auroral arcs were found to have scale sizes in the range $200 \mathrm{~m}$ to $1 \mathrm{~km}$ with a preferred thickness of $400-500 \mathrm{~m}$. The average width of black auroral arcs, $615 \mathrm{~m}$, were similar to the average $(740 \mathrm{~m})$ of the widths of bright auroral structures reported by Maggs and Davis (1968).
Karlsson and Marklund (1996) observed intense electric fields, assumed to be diverging, at low altitude (1400$1770 \mathrm{~km}$ ) and found that their scale sizes were inversely proportional to the intensities of the electric fields, with the most intense electric fields being those with the smallest scale size. The typical scale size of the most intense of these electric field structures was $1-5 \mathrm{~km}$. This inverse relation was also found in a Cluster study (Johansson et al., 2005), including both converging and diverging electric fields. An inverse proportionality has also been found between the scale size and intensity of FACs in the auroral region (Stasiewicz and Potemra, 1998). The most intense current magnitudes were found for the the smallest $\sim 100 \mathrm{~m}$ sized currents, and the intensity dropped with increasing scale size up to the largest FAC widths $(\sim 500 \mathrm{~km})$.

Alfvén waves are also capable of accelerating electrons and powering the aurora, as shown by, e.g., Keiling et al. (2003). Chaston et al. (2003) discussed Alfvén wave driven arcs. The widths of those arcs were of the order of $1 \mathrm{~km}$ at ionospheric altitude. Smaller arcs could possibly be produced but they would have a too low intensity to explain the arcs of some $100 \mathrm{~m}$ observed by Maggs and Davis (1968). In a statistical study of upward accelerated electrons, Andersson and Ergun (2006) found good agreement between the electric field measurements and the characteristic energy of electrons in 50 percent of the events, consistent with the quasi-static model. Andersson and Ergun (2006) speculated that the poor correlation for the other half of the events might be due to Alfvén wave acceleration of the electrons. Another explanation would be that the electric field sometimes partly extends down to the ionosphere, giving a mismatch between the measured electric field and the up-going electrons. Examples of this have been found by Hwang et al. (2006). In a study of downward electron acceleration, Newell et al. (1996) found, using the DMSP satellites, that the widths of such regions had a exponential distribution with a characteristic width of $28-35 \mathrm{~km}$.

In this study, the scale sizes, $S(E)$, of 797 intense auroral electric field structures observed by the Cluster satellites at geocentric distances of 4-7 $R_{E}$ are calculated together with the scale sizes of the associated field-aligned currents, $S$ (FAC), and density gradients, $S(d n)$. The electric field scale sizes are investigated with respect to various conditions (e.g. summer/winter) and in relation to $S(\mathrm{FAC}), S(d n)$ and other plasma parameters. Monopolar electric fields (Sshaped potential structures) and bipolar electric fields (Ushaped potential structures) have been identified and, for the latter ones, a determination of whether they are diverging or converging has been conducted.

\section{Method}

The electric fields investigated in this statistical study were measured by the Cluster EFW instrument (Gustafsson et al., 
1997). A database was created by selecting events at geocentric distances between 4 and $7 R_{E}$ where the magnitude of the electric fields, when mapped to ionosphere, exceeded $0.15 \mathrm{~V} / \mathrm{m}$. This database was also used by Johansson et al. (2005) in a study of the characteristics and occurrence of intense high-altitude electric fields. A total of 797 events have been used in this study. For each of the events, $S(E)$ was calculated as the full-width half-maximum of the peak value. This rather simple method was also used by Karlsson and Marklund (1996) and also in other types of scale size determinations (e.g. Knudsen et al., 2001). The differences in definitions of scale sizes and type of measurement must be kept in mind when the results are compared.

The potential is calculated over a region proportional to the scale size, $S(E)$, by integrating the perpendicular (to the background magnetic field) electric field. Then a smaller region (still proportional to $S(E)$ ) is extracted. The first point in this new, shorter interval is chosen as reference point (zero level). Different types of electric fields have been separated by applying criteria to the potential. The potential signature of a monopolar electric field is a step, while converging and diverging electric fields are associated with negative ("valley") and positive ("hill") signatures in the potential, respectively. By comparing the potential (calculated from the electric field) mid-value and start and end values in a region centered at the electric field peak, the criteria could be checked automatically. An event where the mid-value of the potential is greater (smaller) than the start value but smaller (greater) than the end value has been labelled monopolar. If the midvalue is greater (smaller) than both start and end values, the event has been labelled diverging (converging) bipolar. The potential variation across the structure has been determined as the maximum or minimum value for bipolar events and as the end value of the shorter region for monopolar events.

A fourth degree polynomial was fitted to the magnetic field measured by the FGM instrument (Balogh et al., 1997) and removed from the measured value. From the residual magnetic field, and using the infinite current sheet approximation, the FACs associated with the electric field structures were calculated. The scale sizes $S$ (FAC) have also been calculated as the full-width half-maximum of the current magnitude at the time of the event. The width of the current region, determined as the region with the same current direction as at the time of the event, has been used as an alternative to $S$ (FAC).

The electric fields studied have been found to be colocated with density gradients, which are important for the shape of the associated potential structure (Johansson et al., 2006). The spatial scale used in this study to characterize the density gradients is the scale size of the density gradient, $S(d n)$. Note that $S(d n)$ is not the scale size of a peak in the density. Using CIS ion data (Rème et al., 1997), the gradient in the density was calculated and the maximum value of the density gradient associated with the electric field event was found. $S(d n)$ was then determined as the full-width half-maximum of that peak in the density gradient. The proton gyroradius and inertial scale length have also been calculated. Note that there are no CIS data available from Cluster 2.

The time resolution of the EFW instrument is, in normal mode, $0.04 \mathrm{~s}$ and the spatial resolution at ionospheric altitudes will be $0.015 \mathrm{~km}$ for measurements at $5 R_{E}$ geocentric distance. The CIS instrument has a time resolution of $4 \mathrm{~s}$ giving mapped spatial resolutions of $0.8-2.0 \mathrm{~km}$ for the geocentric distances in this study $\left(4-7 R_{E}\right)$. The FGM magnetic field data used have a spin resolution, same as the CIS instrument.

The electric field magnitudes and the spatial scales have been mapped to the ionospheric altitude for reference using the dipole magnetic field approximation. The mapping to the equator in Fig. 4 has been done using the same approximation (Mozer, 1970; Weimer and Gurnett, 1993).

\section{Scale size observations}

The calculated electric field scale sizes $S(E)$ have been compared with the scale sizes of the associated field-aligned currents $S$ (FAC), and density gradients, $S(d n)$. The relation of $S(E)$ to the proton gyroradius and the proton inertial length, and the variation of $S(E)$ with different parameters, season and $K_{p}$, have also been investigated. All values are mapped down to ionospheric altitude.

\subsection{Typical scale sizes}

Figure 1 displays the number of events versus $S(E), S(d n)$ and $S($ FAC) in the form of histograms, where black lines represent monopolar events, red and blue lines represent converging and diverging bipolar events, respectively. Each bin is $1 \mathrm{~km}$ wide. FAC width, proton gyroradius and proton inertial length are presented in the same form in Fig. 2. Note the extended range of the $\mathrm{x}$-axis and that each bin in Fig. 2 is $4 \mathrm{~km}$ wide. Tables 1 and 2 give typical scale sizes, mean and median values, variance, skewness, 95th percentiles and the total number of events for the different types of electric fields. Mean and median values are given together with errors using the $95.5 \%$ confidence interval. "Typical" scale size or value refers to the most populated bin in Figs. 1 and 2. All spatial values are mapped to the ionosphere for reference. Skewness is a measure of the asymmetry of a distribution. All distributions in this study have positive skewness, i.e., the right tail (large scale sizes) is more pronounced than the left tail (small scale sizes). The higher the skewness, the more asymmetric the distribution.

It is seen in Tables 1 and 2, and in Figs. 1 and 2 that a majority of the events are monopolar and that there is an almost equal amount of converging and diverging bipolar electric field events. The $S(E)$ distributions (top panel in Fig. 1) are similar for bipolar and monopolar events, and they have the same typical scale size $(4-5 \mathrm{~km})$. It can also be seen that the scale size distribution of the monopolar structures 

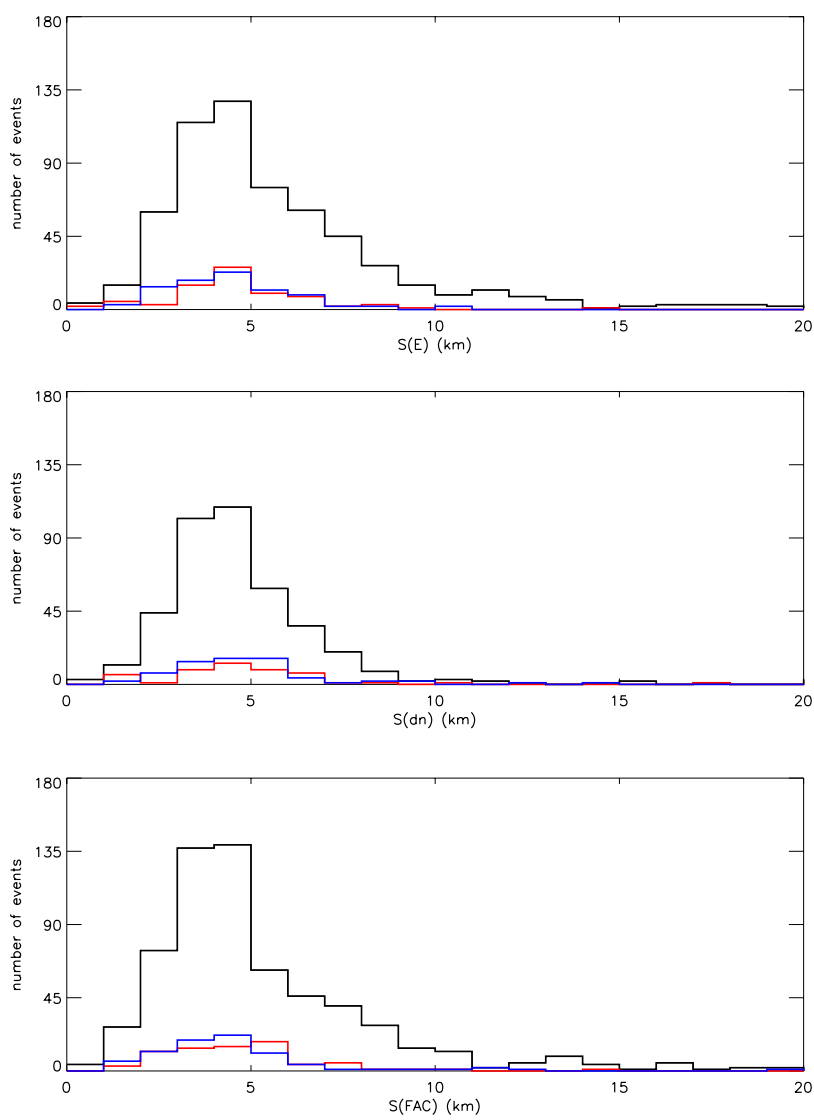

Fig. 1. The number of events versus $S(E), S(d n)$, and $S($ FAC), black lines represent monopolar events while red and blue lines represent converging and diverging bipolar events, respectively.

is more asymmetric than the ones for bipolar structures. This is reflected in a higher median value and a larger skewness. There are more diverging than converging electric fields with scale sizes $<4 \mathrm{~km}$ but otherwise no clear difference can be observed between these two types of bipolar electric fields.

The median scale sizes for the electric field, FAC and density gradient are all in the range $4.2-4.9 \mathrm{~km}$. This is true for both monopolar and bipolar events in all three parameters but the errors are larger for the bipolar events. The distributions of these scale sizes are also similar, and the number of events drops rather quickly as the scale size increases. For all three parameters, the drop is less steep for the monopolar events. This can be seen in a higher skewness for the monopolar events. The median values of $S(E), S(\mathrm{FAC})$ and $S(d n)$ are smaller for the diverging events compared to the converging events but generally, diverging and converging bipolar events have similar $S(E), S(d n)$ and $S(\mathrm{FAC})$ distributions.

The FAC widths have a median width of $9.7 \mathrm{~km}$ for monopolar electric fields and 8.3 (9.5) km for diverging (converging) bipolar electric fields, larger than the scale sizes of the electric field. (The uncertainty in FAC widths for diverging events is large.) The distribution of the FAC widths (top
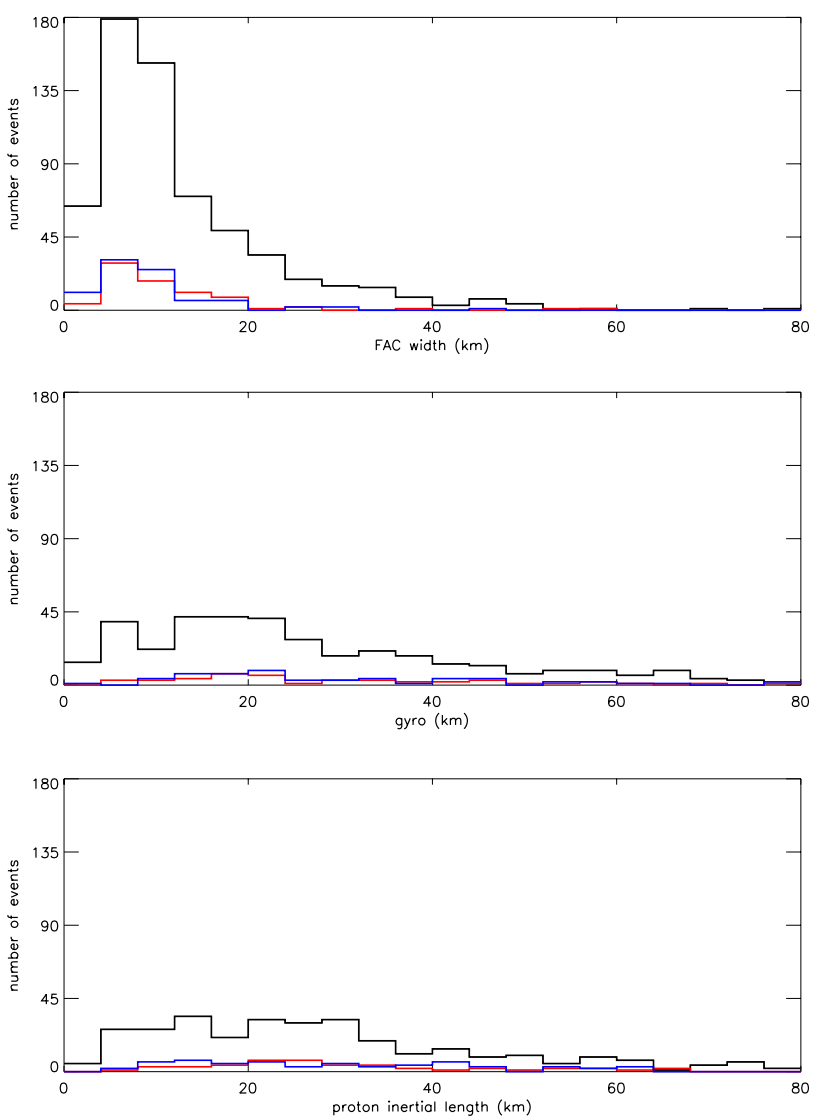

Fig. 2. The number of events versus FAC width, proton gyroradius and proton inertial length, black lines represent monopolar events while red and blue lines represent converging and diverging bipolar events, respectively. Note the increased range on the x-axis (compared to Fig. 1).

panel in Fig. 2) resembles the distributions in Fig. 1 but extend into grater values. This shows that the electric field structures are embedded in the FAC regions. (Remember that $S($ FAC) is the full-width half-maximum of the current magnitude and FAC width is the the region with the same current direction.)

The proton gyroradius (middle panel in Fig. 2) and the proton inertial length have also been calculated (bottom panel in Fig. 2). The populations from which these parameters are calculated are typically dominated by hot magnetospheric ions with a perpendicular temperature of a few $\mathrm{keV}$.

The proton gyroradius has a different distribution, being larger than the previous scale sizes. The peak in the number of monopolar events is broad, ranging from $4 \mathrm{~km}$ (similar to the typical values in $S(E), S(\mathrm{FAC})$ and $S(d n))$ to $24 \mathrm{~km}$. The median value is $22.3 \mathrm{~km}$, so almost half of the events are associated with proton gyroradii outside of the broad peak region. The rather flat distribution is reflected in a low skewness. The bipolar events have median proton gyroradii of $25.7 \mathrm{~km}$ (diverging) and $23.4 \mathrm{~km}$ (converging). 

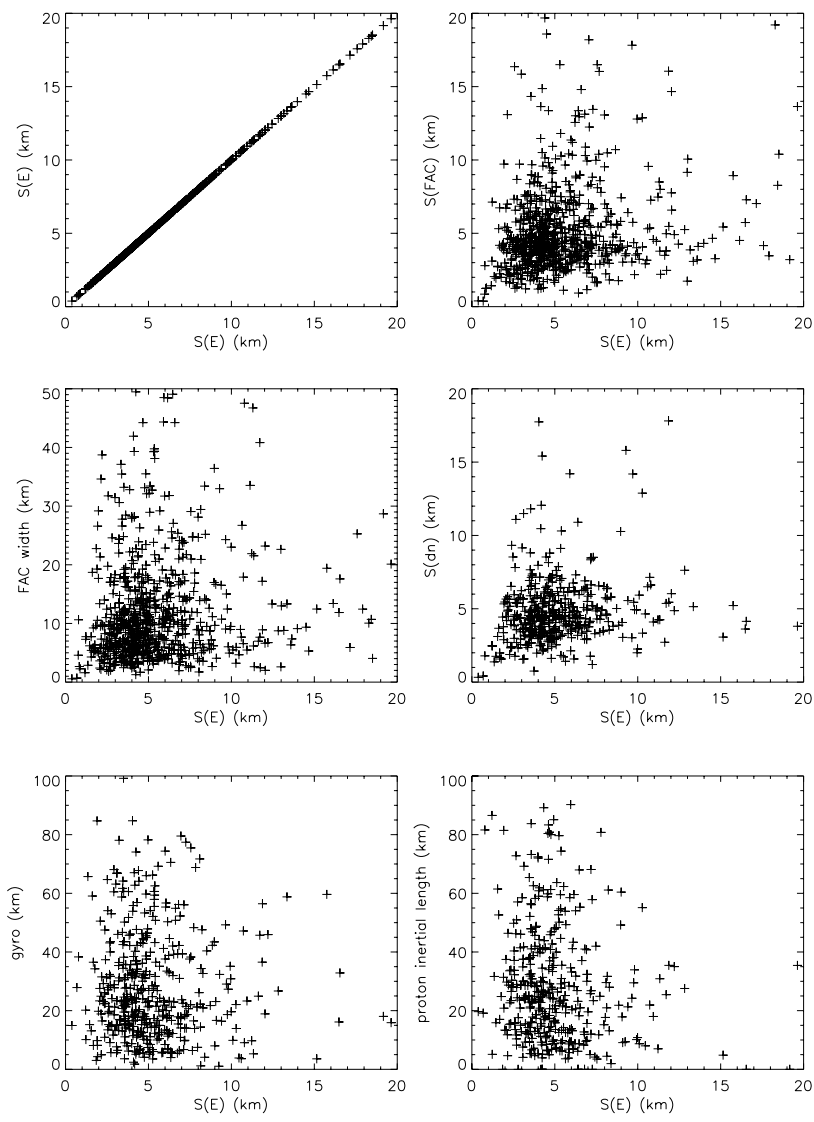

Fig. 3. Overview of all events in the form of scatter plots. The parameters plotted are $S(E), S(\mathrm{FAC})$, FAC width, $S(d n)$, proton gyroradii and proton inertial length, all vs. $S(E)$. These values are mapped to the ionosphere.

The proton inertial lengths have a distribution similar to the gyroradius distribution. The median values are slightly greater while the typical proton inertial lengths for monopolar events overlap the range of typical gyroradii.

As was shown in Figs. 1 and 2, and in Tables 1 and 2, the typical, mean and median scale sizes of the electric field were similar to those of the FACs and density gradients. Another way to describe the relation between the different parameters is to present the data in the form of scatter plots. Figures 3 and 4 are such plots with the parameters plotted versus $S(E)$. In Fig. 3, a general trend of increasing $S(d n)$, $S($ FAC) and FAC width for increasing $S(E)$ can be observed. The proton gyroradii and proton inertial length appear independent of $S(E)$. The correlation coefficients are low, below 0.2 for all pairs. However, if the values instead are mapped to the equatorial plane, as done in Fig. 4, it is seen that also these two parameters correlate well with $S(E)$. For $S(d n)$, $S(F A C)$ and FAC width, the correlation with $S(E)$ is more clearly observed. The correlation coefficients between $S(E)$ and the five other spatial scales, mapped to the equatorial plane, are for all pairs above 0.9 except for $S(E)$ and proton
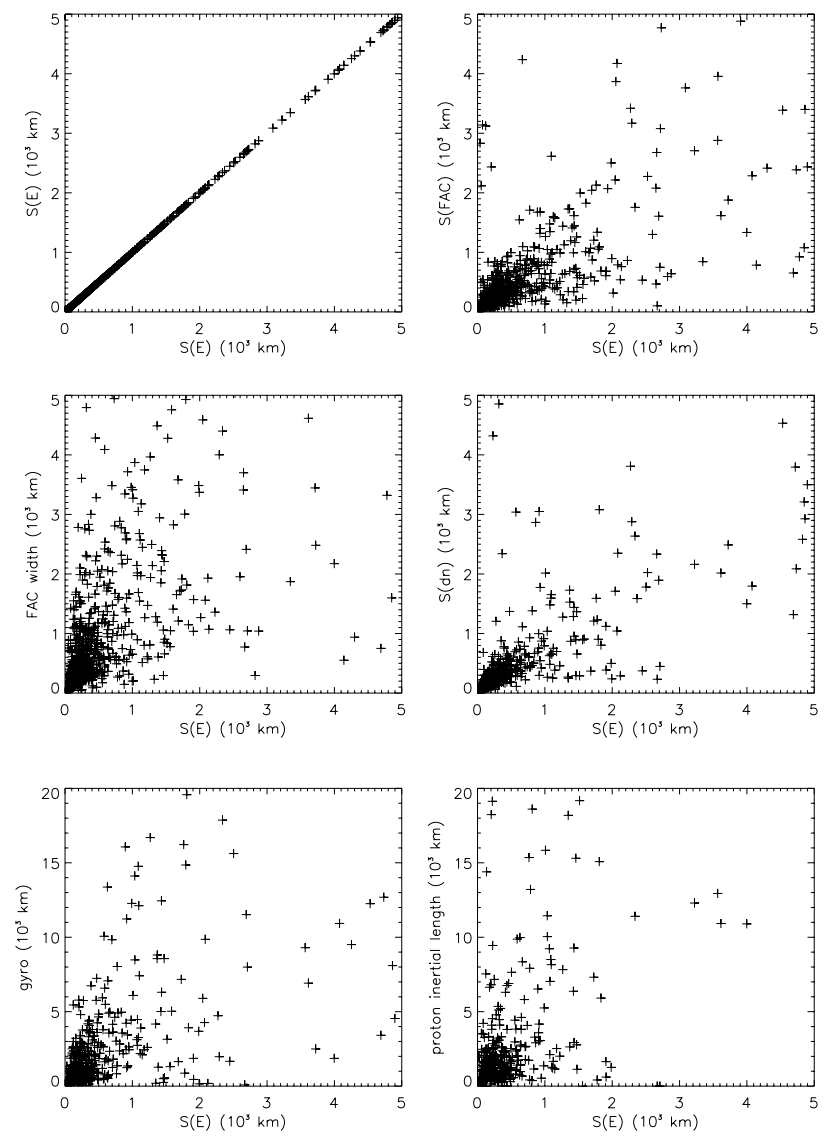

Fig. 4. Similar to Figure 3 but this time mapping to the equatorial plane.

gyroradius where the correlation coefficient is 0.8. (When the scale sizes are mapped to the ionosphere or to the equatorial plane, the spread in latitude and altitude give different mapping factors for each event, hence the relationship between the events in the two scatter plots can be different.) The improved correlation in the equatorial plane might indicate that the mechanism determining the scale sizes observed at Cluster's altitude is located between the region of observation and the equatorial plane.

\subsection{Characteristics of $S(E)$}

The influence of season and geomagnetic activity (as measured by the $K_{p}$ index) and possible dependence on MLT and CGLat have been investigated. How $S(E)$ is related to the electric field magnitude and the potential variation across the structure have also been considered.

\subsubsection{Influence of geomagnetic activity and season}

In the upper two panels in Fig. 5, the (monopolar and bipolar) events have been divided into four subsets. Events during summer and winter (periods of three months) have been 
Table 1. Some statistical properties for the electric field scale size, $S(E)$, and the field-aligned current scale size, $S$ (FAC), and the FAC width. The results are separated for different types of electric fields. The values are mapped down to ionospheric altitude for reference and given in km. 11 events could neither be labelled monopolar nor bipolar by the routine used. For median and mean values, errors using the $95.5 \%$ confidence interval are given.

\begin{tabular}{lccc}
\hline & monopolar & bipolar, diverging & bipolar, converging \\
\hline Number of events & 625 & 76 & 85 \\
\hline$S(E):$ & & & \\
\hline typical & $4-5$ & $4-5$ & $4-5$ \\
mean & $6.9 \pm 0.57$ & $4.5 \pm 0.41$ & $4.7 \pm 0.45$ \\
median & $4.9 \pm 0.71$ & $4.2 \pm 0.51$ & $4.6 \pm 0.56$ \\
variance & 50.7 & 3.15 & 4.22 \\
skewness & 4.2 & 0.87 & 1.50 \\
95th percentile & 19.6 & 7.2 & 7.2 \\
\hline$S($ FAC): & & & \\
\hline typical & $4-5$ & $4-5$ & $5-6$ \\
mean & $6.3 \pm 0.86$ & $8.5 \pm 5.6$ & $6.2 \pm 2.6$ \\
median & $4.4 \pm 1.1$ & $4.3 \pm 7.0$ & $4.7 \pm 3.3$ \\
variance & 114.3 & 592.4 & 149.5 \\
skewness & 10.4 & 6.5 & 8.0 \\
95th percentile & 13.1 & 12.9 & 9.6 \\
\hline FAC width: & & & \\
\hline typical & & & $4-8$ \\
mean & $4-8$ & $4-8$ & $11.7 \pm 2.1$ \\
median & $13.7 \pm 1.4$ & $12.8 \pm 7.1$ & $9.5 \pm 2.6$ \\
variance & $9.7 \pm 1.8$ & $8.3 \pm 8.9$ & 27.9 \\
skewness & 309.7 & 967.8 & \\
95th percentile & 9.2 & 8.2 & \\
\hline
\end{tabular}

separated after the level of geomagnetic activity under which they occur. The $K_{p}$ index has been used to identify high $\left(K_{p}>3\right)$ and low $\left(K_{p} \leq 3\right)$ activity events.

When comparing high activity events occurring during summer (solid line in the top left panel) with low activity events occurring during winter (dotted line in the top right panel), an increase in $S(E)$ can be observed for the latter events. The typical scale size increases from $2-3 \mathrm{~km}$ to 4 $5 \mathrm{~km}$ and the distribution of events is also shifted towards greater $S(E)$ for the low activity winter events compared to the high activity summer events. During the former times, both the background conductivity and the particle induced conductivity are lower than during the high activity summer events. This indicates that the ionospheric conductivity influences the scale sizes of auroral structures as observed at high altitude in the form of intense electric fields. The typical scale sizes are $3-4 \mathrm{~km}$ when $K_{p}>3$ during winter months (solid line in the upper right panel) and $4-5 \mathrm{~km}$ when $K_{p} \leq 3$ during summer months (dotted line in the upper left panel). The mean and median values for the different subsets are given in Table 3 together with errors indicating the 95.5\% confidence interval. The highest mean and median values
(6.9 and $5.1 \mathrm{~km}$, respectively) are found for summer months during low activity while the lowest median value $(3.6 \mathrm{~km})$ is found for summer months during high activity and the lowest mean value $(4.5 \mathrm{~km})$ is found for winter months during high activity. There appear to be both a season and an activity dependence of which the latter is more important. The number of events in each of these four subsets are rather small. There are only 22 high $K_{p}$ events during summer, which gives some uncertainty to the mean and median values for this subset.

The middle and lower panels in Fig. 5 display the number of events for bins in $K_{p}$ and the average electric field magnitude. The color coding is the same as in Fig. 1. Most events are found for medium activity $\left(K_{p}=1-4\right)$ but Johansson et al. (2005) showed a weak increase in the probability of finding an event with increasing $K_{p}$. The average electric field magnitude (plotted together with error bars indicating the $95.5 \%$ confidence interval) is found to increase with higher geomagnetic activity (except from the highest $K_{p}$ values) consistent with the inverse relation between $S(E)$ and the electric field magnitude (see below). The average electric field magnitudes of diverging (blue line) and converging (red line) bipolar and monopolar (black line) events are similar. 
Table 2. Same kind of table as Table 1 but this time for the scale sizes of the density gradients, $S(d n)$, and the proton gyroradii and the inertial proton scale. For median and mean values, errors using the $95.5 \%$ confidence interval are given.

\begin{tabular}{lccc}
\hline & monopolar & bipolar, diverging & bipolar, converging \\
\hline Number of events & 625 & 76 & 85 \\
\hline$S(d n):$ & & & \\
\hline typical & $4-5$ & $4-5$ & $4-6$ \\
mean & $6.4 \pm 1.1$ & $5.0 \pm 0.52$ & $5.6 \pm 1.2$ \\
median & $4.4 \pm 1.4$ & $4.5 \pm 0.65$ & $4.9 \pm 1.5$ \\
variance & 196.0 & 5.12 & 29.3 \\
skewness & 9.20 & 1.80 & 4.78 \\
95th percentiles & 10.3 & 9.3 & 10.3 \\
\hline gyroradius: & & & $20-24$ \\
\hline typical & $12-20$ & $16-20$ & $30.9 \pm 4.2$ \\
mean & $26.5 \pm 1.4$ & $31.4 \pm 4.4$ & $23.4 \pm 5.3$ \\
median & $22.3 \pm 1.8$ & $25.7 \pm 5.5$ & 382.7 \\
variance & 325.2 & 362.4 & 0.90 \\
skewness & 0.94 & 1.01 & 69.2 \\
95th percentile & 64.1 & 78.2 & 1.6 \\
\hline inertial proton length: & & & 81.6 \\
\hline typical & $12-16$ & $20-28$ & $29.1 \pm 6.7$ \\
mean & $84.5 \pm 10.4$ & $46.8 \pm 14.1$ & \\
median & $30.8 \pm 13.0$ & $31.7 \pm 17.6$ & 3753.5 \\
variance & 16794.8 & 4.1 & \\
skewness & 2.8 & 136.9 & \\
95th percentile & 356.3 & & \\
\hline
\end{tabular}

Table 3. Mean and median scale sizes in km for the electric field, $S(E)$, separated for summer and winter, and high $(>3)$ and low $(\leq 3) K_{p}$. The values are mapped down to ionospheric altitude for reference and given in $\mathrm{km}$. For median and mean values, errors using the $95.5 \%$ confidence interval are given.

\begin{tabular}{ccccc}
\hline & summer, high $K_{p}$ & summer, low $K_{p}$ & winter, high $K_{p}$ & winter, low $K_{p}$ \\
\hline Number of events & 22 & 82 & 49 & 92 \\
\hline mean & $4.7 \pm 2.0$ & $6.9 \pm 1.3$ & $4.5 \pm 0.61$ & $5.6 \pm 0.90$ \\
median & $3.6 \pm 2.5$ & $5.1 \pm 1.7$ & $4.0 \pm 0.76$ & $4.4 \pm 1.2$ \\
\hline
\end{tabular}

There are only two events in the bin for $K_{p} 6$ and the error is large, so the result in this bin is not reliable.

\subsubsection{Potential and electric field magnitude}

The potential variation across the electric field structure has been calculated for each event and is presented in the upper panels in Figs. 6 (monopolar events) and 7 (bipolar events), for bins in $S(E)$. For $S(E)>1 \mathrm{~km}$, a weak increase in the potential with increasing $S(E)$ is seen, from $\sim 0.9 \mathrm{kV}$ to $\sim 2.9 \mathrm{kV}$ for scale sizes of $9 \mathrm{~km}$. This calculated potential is more reliable than the potential estimation made by Johansson et al. (2005) and confirms the trend of an increasing potential with scale size, seen in that work. A peak in the po- tential is found for $S(E)=11 \mathrm{~km}$ and up to $S(E)=14 \mathrm{~km}$, with a maximum value of close to $6 \mathrm{kV}$. It is interesting that this scale size region corresponds to scale sizes of electric field structures observed in rocket measurements of arc associated electric fields (Marklund et al., 1982) and close to the typical arc widths observed by Knudsen et al. (2001).

For the bipolar events, a decrease in the potential variation across the electric field structures is observed, contrary to what was seen for monopolar events. The converging and diverging events have maxima of $4.6 \mathrm{kV}$ and $3.5 \mathrm{kV}$, respectively, before reaching rather constant values at approximately $2.0 \mathrm{kV}$ and $1.4 \mathrm{kV}$, respectively. However, as was seen in Fig. 1, the number of events in the bipolar distributions are rather few outside the peaks, raising uncertainties 

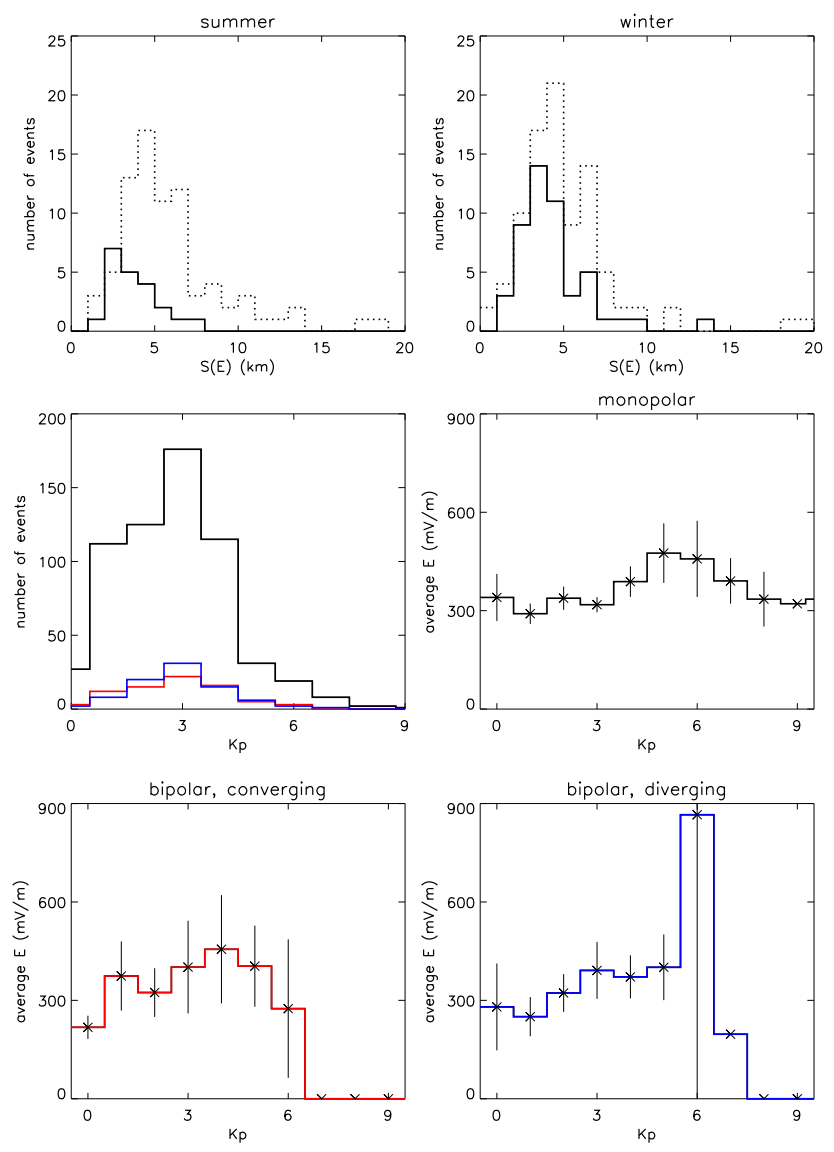

Fig. 5. Upper panels: Number of events vs. $S(E)$ for high $\left(K_{p}>3\right.$, solid line) and low ( $K_{p} \leq 3$, dotted line) geomagnetic activity and summer (left) and winter (right). Each season is defined as a three month period. Middle and lower panels: Number of events vs. $K_{p}$ and average electric field magnitude for bins in $K_{p}$, events separated for monopolar (black line) and diverging (blue line) and converging (red line) bipolar electric fields. The error bars indicate the $95.5 \%$ confidence interval. If no error bar is indicated, then this bin contains no or only one event.

in the reliability of the results in the ends of the distributions. This can also be seen from the large error bars for most of the bins.

Figures 6 and 7 also display the distribution of events as a function of the electric field magnitude (bottom panels). The general trend is the same for monopolar and bipolar events. The typical magnitude is found to be close to $250 \mathrm{mV} / \mathrm{m}$. For electric field magnitudes greater than $600 \mathrm{mV} / \mathrm{m}$, the number of events decreases rapidly. Remember that the cutoff at $150 \mathrm{mV} / \mathrm{m}$ is due to the selection criterion when compiling the database.

The average electric field magnitude is plotted versus $S(E)$ in the middle panels and is seen to be decreasing with increasing $S(E)$ for both monopolar and bipolar events (ignore the last monopolar bin, with a large average electric field, since it contains only one event). This means that a
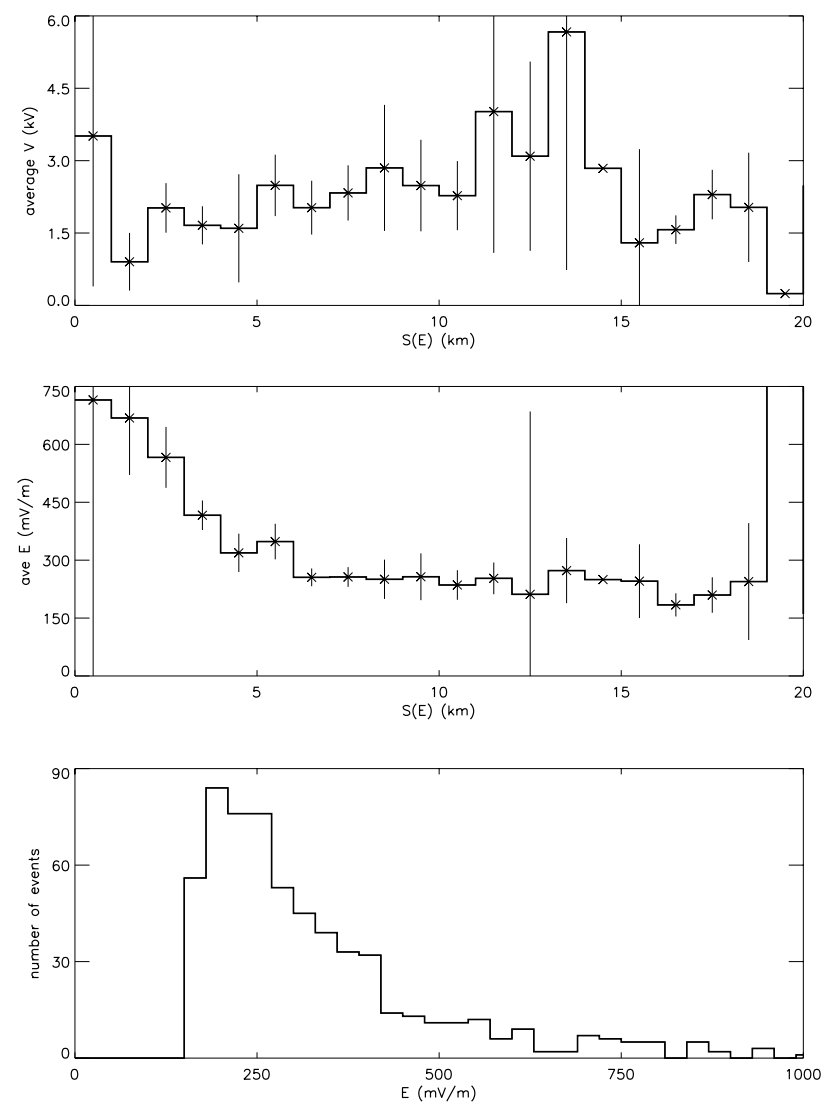

Fig. 6. Average potential $V$ (upper panel) and average electric field magnitude (middle panel) for bins in $S(E)$ together with number of events vs. electric field magnitude (bottom panel). Only monopolar events. All values mapped to the ionosphere. A selection criterion in this study was an electric field magnitude of at least $150 \mathrm{mV} / \mathrm{m}$. The error bars indicate the $95.5 \%$ confidence interval.

smaller scale structure is likely to be more intense than a larger scale structure. Since the average potential across the monopolar structures increases with increasing scale size, the decrease in magnitude must be slower than the increase in scale size. This is only true for monopolar events. Among the bipolar electric fields, the converging ones are typically more intense than the diverging ones but the difference is small.

\subsection{Related field-aligned currents}

Lyons et al. (1979) observed a relation between the parallel current density and the parallel potential drop in the upward current region. The ratio $G_{\mathrm{up}}=j_{\|} / V_{\|}$is known as the LyonsEvans-Lundin constant. This can be compared with the theoretically derived Knight relation (Knight, 1973) which in its linear regime can be written as $j_{\|}=K \Delta \Phi_{\|}$where $K$ is the Knight conductance. The potentials, $V_{\perp}$, determined from integrations of the perpendicular electric fields in this study have been used together with the calculated field-aligned 

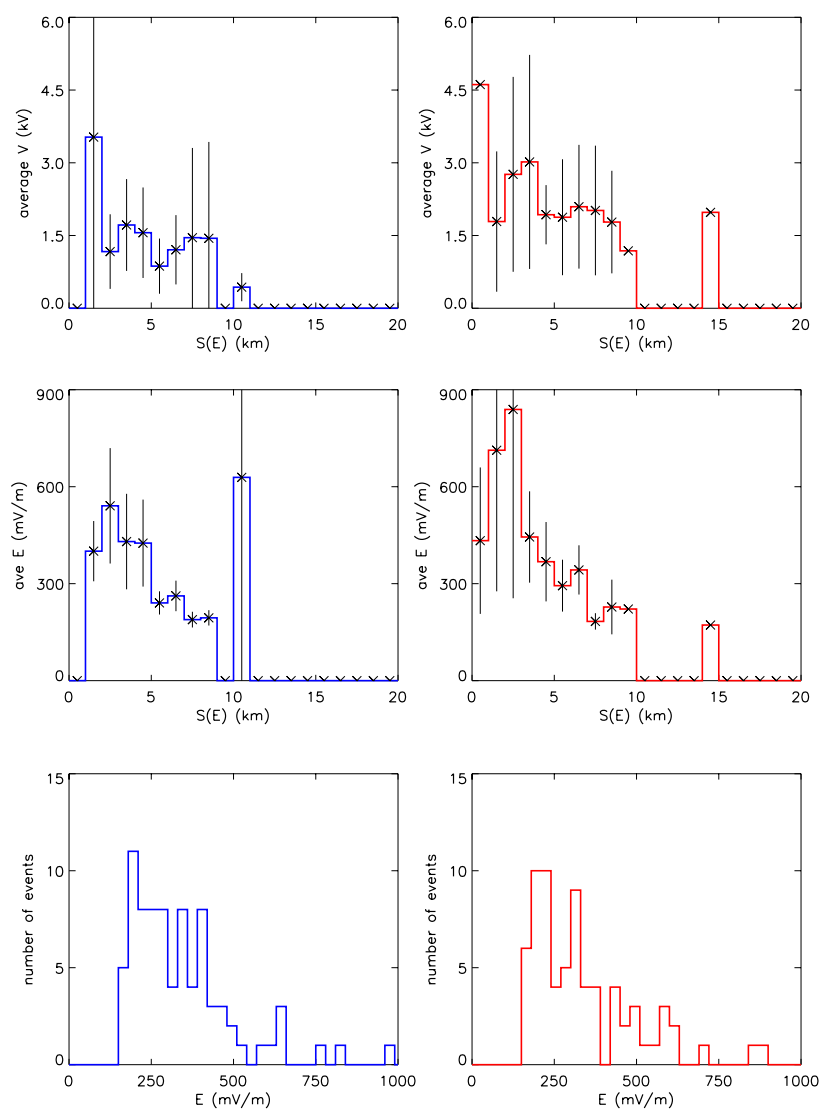

Fig. 7. Same as Figure 6, but this time only converging (red) and diverging (blue) bipolar events. The error bars indicate the $95.5 \%$ confidence interval.

current, $j_{z}$, to obtain a kind of proxy for the Lyons-EvansLundin constant (assuming $V_{\perp}=V_{\|}$). Only one value is calculated for each event. The results are found to be between $10^{-9}-10^{-8} \mathrm{~S} / \mathrm{m}^{2}$, within the range of reported results of the Lyons-Evans-Lundin constant, $10^{-10}-10^{-8} \mathrm{~S} / \mathrm{m}^{2}$ (Elphic et al., 1998).

Figure 8 displays the FAC intensity as a function of FAC width, with values mapped to the ionosphere. Events where the intensity is small are found for all FAC widths. However, the most intense FACs are associated with smaller FAC widths, and the number of intense FACs decreases with increasing FAC width. In this regard, the upward and downward FACs behave in the same way. This has also been observed by Peria et al. (2000). This characteristic is similar to the relation between the electric field magnitude and $S(E)$. In both cases, an inverse proportionality to the spatial scale is observed. It is also worth noticing that the widths of upward and downward FACs are similar.

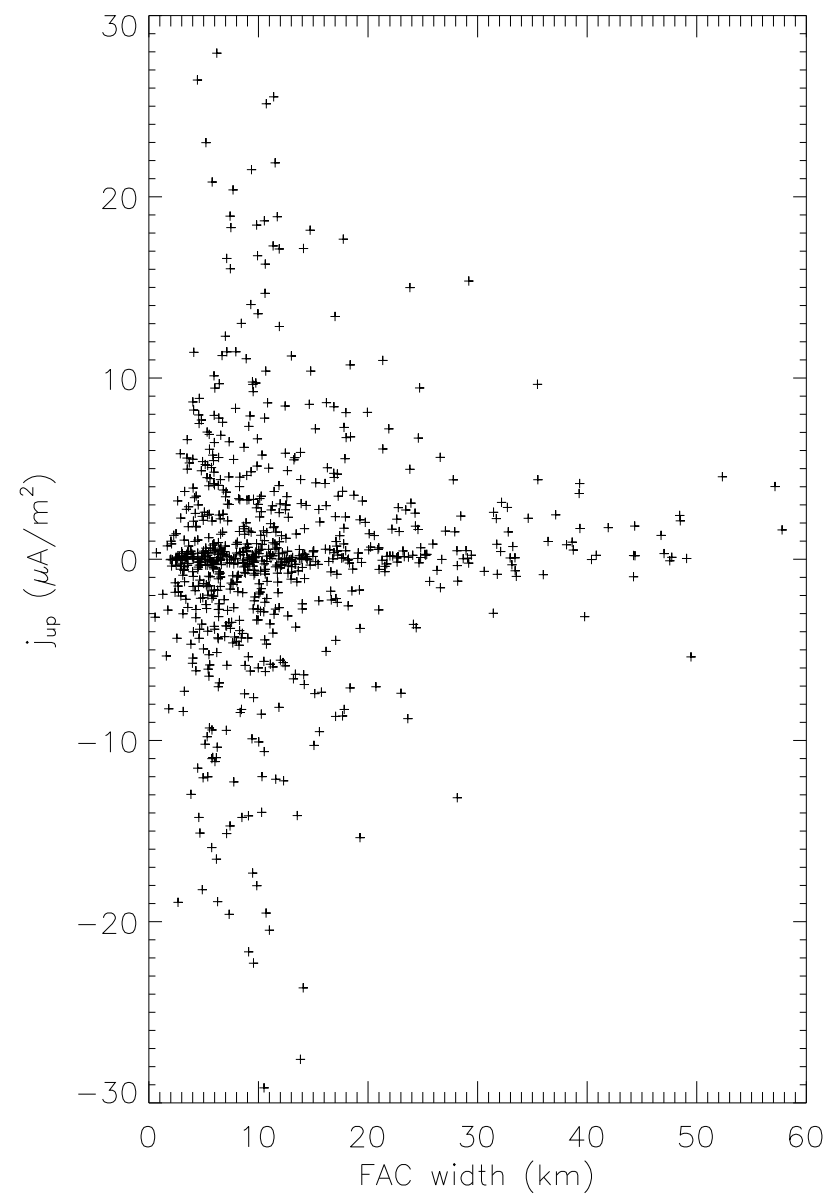

Fig. 8. Scatter plot of the FAC magnitude vs. FAC width, both mapped to the ionosphere. Positive values are upward FAC.

\section{Discussion}

An important question in auroral physics is how to explain the discrepancies in scale sizes between, on one hand, reported optical observations, and on the other hand, theoretically predicted scales and in situ measurements, prevailing for both bright and black aurora. It is possible that the highaltitude structures, by some mechanism, are divided into several, narrower structures. This concept is illustrated by the results in McFadden et al. (1999), although their smaller scales were an order of magnitude larger than the fine-scale auroral arcs. The work presented here focuses on the scale sizes of intense auroral electric fields observed by Cluster at high altitude (4-7 $R_{E}$ geocentric distance). Electric fields with intensities (mapped to the ionosphere) less than $150 \mathrm{mV} / \mathrm{m}$ are not included. Based on earlier event studies (e.g., Johansson et al., 2004) it is assumed that most of the events have a quasi-static structure.

The typical scale sizes of the studied intense electric fields (both monopolar and bipolar) are, when mapped to ionospheric altitude, $4-5 \mathrm{~km}$, with a median scale size of $4.8 \mathrm{~km}$. 


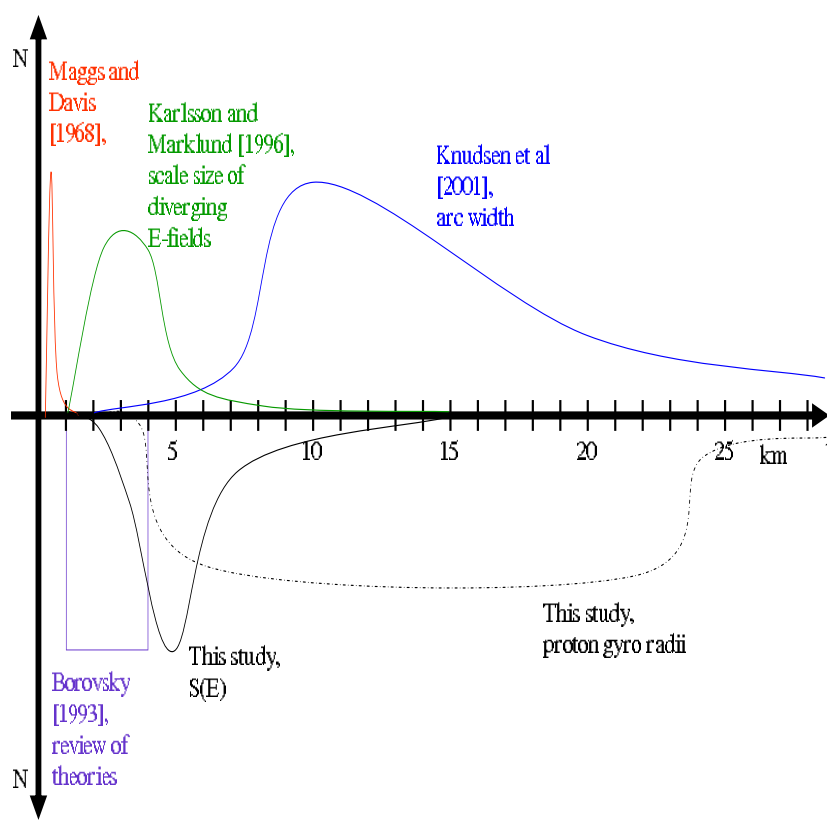

Fig. 9. A sketch summarizing some auroral scale size observations. The optical measurements of arc widths by Maggs and Davis (1968) and Knudsen et al. (2001) are shown in red and blue, respectively. The green line indicates the scale sizes for diverging bipolar electric fields observed by Freja (Karlsson and Marklund, 1996). A majority of the theories reviewed by Borovsky (1993) predicted scale sizes in the range $1-4 \mathrm{~km}$; they are here represented by the purple rectangle. Results from this study are the solid black line, $S(E)$, and the dashed-dotted black line, proton gyroradii.

This is somewhat larger than, but in rough agreement with, the theoretical predictions reviewed by Borovsky (1993). These are scale sizes in the gap of the arc width distribution of the compiled studies by Maggs and Davis (1968) and Knudsen et al. (2001). The largest scale sizes in this study overlap with the small side tail of the meso-scale arcs widths in Knudsen et al. (2001). Figure 9 tries to summarize these different observations of auroral scales. The distributions of the fine-scale and meso-scale auroral arcs observed by Maggs and Davis (1968) and Knudsen et al. (2001) (red and blue lines) are outlined. Typical electric field scale sizes observed by Cluster (this study, solid black line) and Freja (Karlsson and Marklund (1996), green line and diverging electric fields only) fall into the gap between these two distributions. Karlsson and Marklund (1996) uses the same method (full-width half-maximum) to determine the scale sizes of the electric field structures as in this study. The median scale size of the diverging electric fields observed in this study is $4.6 \mathrm{~km}$, within the range of typical scale sizes (1$5 \mathrm{~km}$ ) observed by Freja. The electric fields investigated in this study are intense ( $\geq 150 \mathrm{mV} / \mathrm{m}$ at ionospheric altitude). Since an inverse relation between intensity and scale size has been found (see Fig. 6), lowering the limit in intensity for event selection would probably result in an increased number of larger scale sizes. Intense auroral electric field structures have been shown to be related to plasma boundaries (Johansson et al., 2006; Marklund et al., 2007), hence the scale sizes of electric field structures related to arc boundaries can be expected to be smaller than the arc widths determined by optical studies. The purple rectangle in Fig. 9 illustrates where most of the theories reviewed by Borovsky (1993) predict the arc widths to be found.

The widths or scale sizes of auroral arcs and structures reported in the literature are influenced by the resolution of the instrument used and how the data is presented. StenbaekNielsen et al. (1998) mentioned, e.g., how the use of logarithmic scales in particle energy and number flux plots can give too-wide scale sizes, compared to what is seen in optical observations. Using wide-angle lenses or narrow-field cameras can influence the scale size results of visible structures, and the choice will or will not give information of the largescale context of the observation (Knudsen et al., 2001). The criterion used by Newell et al. (1996) selected events where the accelerated electron energy was 3-4 times larger than the source population thermal energy. This type of electron acceleration events, with a characteristic width of $28-35 \mathrm{~km}$, was suggested to correspond to a cluster of individual arcs. The finest arc structures could not be resolved. The resolution of the electric field measurements in this study is good enough to give confidence to the decrease in the $S(E)$ distribution on the small scale side. The electric fields investigated are intense and therefore biased towards smaller scales (or so do the results indicate, see Fig. 6).

As discussed by Weimer et al. (1985), there is a critical scale size (proportional to the electron density and inversely proportional to the height-integrated Pedersen conductivity and the square root of the thermal energy), separating electric fields at high altitude that map down to the ionosphere and those that do not. Since it is the electric fields with scale sizes less than the critical value that do not map, implying parallel electric fields and particle acceleration, the electric fields in this study, which are small scale, are likely to not map down to the ionosphere. They should also correspond to auroral arcs. In earlier Cluster event studies of these kind of electric fields, the potential (calculated from the electric field) and the characteristic energy of up-going particles have been found to roughly agree (Johansson et al., 2004; Figueiredo et al., 2005), indicating non-mapping. For typical values of the input parameters, Weimer et al. (1985) give the critical scale sizes as $80 \mathrm{~km}$, at ionospheric altitude. However, this critical value will vary and might at times be smaller than $80 \mathrm{~km}$. The shape of the auroral structures also influences how the electric field is mapped, or, equivalently, how the potential structures are closed. Spirals and folds have been shown to be associated with non-mapping electric fields, while sheet-like structures (stable arcs), were shown to partly extend down to the ionosphere (Hwang et al., 2006). Future studies considering this subject are planned.

The distribution of the proton gyroradii for the events in this study is sketched as a dashed-dotted line in Fig. 9, clearly 
separated from most other distributions. The proton gyroradii distribution overlaps the arc widths reported by Knudsen et al. (2001). The FAC and density gradient scale size distributions of this study are omitted since they are similar to the electric field scale size distribution.

Two parameters connected to Alfvénic auroral activity are the electron inertial length, $\lambda_{e}$, and the Alfvén wave resistive scale, $\lambda_{A}$. Filamentary Alfvénic field-aligned currents above the aurora with widths similar to $2 \pi \lambda_{e}$ have been observed by FAST (Chaston et al., 2007). The electron inertial length has been calculated (and mapped to ionospheric altitude) for the events in this study. They were typically less then $0.75 \mathrm{~km}$, so $2 \pi \lambda_{e}$ would be $\sim 4.7 \mathrm{~km}$. The widths of the currents associated with the intense electric fields of this study are typically larger, with median values of $8.3-9.7 \mathrm{~km}$, for the different types of electric fields (see Table 1). The other parameter, $\lambda_{A}$, is typically of the order of $10 \mathrm{~km}$ (Pilipenko et al., 2004), and well separated from the typical scale sizes of the intense electric fields investigated here.

The inverse relationship between electric field scale size and magnitude earlier reported from Freja (Karlsson and Marklund, 1996) and Cluster observations (Johansson et al., 2005), can be confirmed in this study. Also, a relationship between electric field scale size and the potential variation across the structure has been found. For the monopolar events, the potential tends to increase with increasing scale size, although the increase is weak. An inverse relationship between FAC width and FAC intensity has also been seen, consistent with earlier results on auroral FACs (Stasiewicz and Potemra, 1998). The FAC widths and intensities of upward and downward currents were found to be similar. A symmetry in the thickness of the current sheets with respect to the polarity was also found by Peria et al. (2000) in a statistical study of FACs derived from FAST data.

The ionospheric conductivity has been shown here to influence the electric field scale size. During periods of high conductivity (summer months and high geomagnetic activity as measured by the $K_{p}$ index), the scale sizes are typically smaller. The dependence on geomagnetic activity is more significant. During periods of high activity, the electric field magnitudes are typically larger (as seen in Fig. 5). This is consistent with the inverse relation between scale size and electric field magnitude. In the downward FAC region, electrons are evacuated from the ionosphere, forming a plasma density hole, as has been shown in simulations (Karlsson and Marklund, 1998). Marklund et al. (2001) showed how a downward current region, to compensate for the depletion, widened so that the total current could remained roughly constant. During this time, the electric field scale size increased as well. This mechanism might explain why the electric field scale sizes in the downward current region are smaller for higher ionospheric conductivities. To maintain a certain current magnitude, a smaller region is required when the conductivity is higher. It is not understood why the same relation is seen for the electric field in the upward current region.

\section{Conclusions}

The main results of this study are:

1. The typical electric field, magnetic field and density gradient scale sizes are all 4-5 km (mapped to ionospheric altitudes), with no clear difference between scale sizes associated with monopolar, converging or diverging bipolar electric fields. However, the scale size distributions for the monopolar events extend to greater values than the bipolar scale size distributions.

2. When mapped to the equatorial plane, $S(d n), S(\mathrm{FAC})$, FAC width, proton gyroradius and proton inertial length correlate well with $S(E)$.

3. The majority of the intense electric field structures (625 events) are monopolar, i.e., associated with S-shaped potential structures. Among the bipolar electric field signatures, associated with U-shaped potential structures, there is approximately an equal number of converging and diverging structures (85 and 76 , respectively).

4. The electric field magnitude is inversely proportional to the scale size while the potential variation across monopolar structures has a small proportionality to the scale size.

5. The most intense field-aligned currents are found for small FAC widths. The widths of upward and downward FACs are similar.

6. Different ionospheric conductivity conditions are reflected in different $S(E)$, as high (low) conductivity favors smaller (larger) scale sizes. Both seasonal variations and geomagnetic activity influence $S(E)$ but the latter is more important. Typical values are $2-3 \mathrm{~km}$ (4$5 \mathrm{~km})$ for $K_{p}>3(\leq 3)$ during summer (winter) months.

Acknowledgements. Work at the Royal Institute of Technology was partially supported by the Swedish National Space Board and the Alfvén Laboratory Centre for Space and Fusion Plasma Physics. S. Liléo acknowledges the support of the Fundação para a Ciência e a Tecnologia (FCT) under the grant SFRH/BD/6211/2001.

Topical Editor I. A. Daglis thanks K. Lynch and another anonymous referee for their help in evaluating this paper.

\section{References}

Andersson, L. and Ergun, R. E.: Acceleration of antiearthward electron fluxes in the auroral region, J. Geophys. Res., 111, A07203, doi:10.1029/2005JA011261, 2006.

Balogh, A., Dunlop, M. W., Cowley, S. W. H., Southwood, D. J., Thomlinsson, J. G., Glassmeier, K. H., Musmann, G., Lühr, H., Buchert, S., Acuna, M., Fairfield, D., Slavin, J., Riedel, W., Schwingenschuh, K., and Kievelson, M.: The Cluster magnetic investigation, Space Sci. Rev., 79/1-2, 65-91, 1997. 
Blixt, E. M. and Kosch, M. J.: Coordinated optical and FAST observations of black aurora, Geophys. Res. Lett., 31, L06813, doi:10.1029/2003GL019244, 2004.

Block, L. P.: Potential double layers in the ionosphere, Cosmic Electrodynamics, 3, 349-376, 1972.

Borovsky, J. E.: Auroral arc thickness as predicted by various theories, J. Geophys. Res., 98, 6101-6138, 1993.

Chaston, C., Peticolas, L., Bonnell, J., Carlson, C., Ergun, R., McFadden, J., and Strangeway, R.: Width and brightness of auroral arcs driven by inertial Alfven waves, J. Geophys. Res., 108, 1091, doi:10.1029/2001JA007537, 2003.

Chaston, C., Hull, A., Bonnell, J., Carlson, C., Ergun, R., Strangeway, R., and McFadden, J.: Large parallel electric fields, currents, and density cavities in dispersive Alfvén waves above the aurora, J. Geophys. Res., 112, A05215, doi:10.1029/2006JA012007, 2007.

Chiu, Y. T. and Schulz, M.: Electrostatic model of a quiet auroral arc, J. Geophys. Res., 83, 629-642, 1978.

Elphic, R. C., Bonnell, J., Strangeway, R., Kepko, L., Ergun, R., McFadden, J., Carlson, C., Peria, W., Cattell, C., Klumper, D., Shelley, E., Peterson, W., Moebius, E., Kistler, L., and Pfaff, P.: The auroral current circuit and field-aligned currents observed by FAST, Geophys. Res. Lett., 25, 2033-2036, 1998.

Figueiredo, S., Marklund, G., Karlsson, T., Johansson, T., Ebihara, Y., Ejiri, M., Ivchenko, N., Lindqvist, P.-A., Nilsson, H., and Fazakerley, A.: Temporal and spatial evolution of discrete auroral arcs as seen by Cluster, Ann. Geophys., 23, 2531-2557, 2005, http://www.ann-geophys.net/23/2531/2005/.

Galperin, Y. I.: Multiple scales in auroral plasmas, J. Atmos. Sol.Terr. Phy., 64, 211-229, 2002.

Gustafsson, G., Boström, R., Holback, B., Holmgren, G., Lundgren, A., Stasiewicz, K., Åhlén, L., Mozer, F., Pankow, D., Harvey, P., Berg, P., Ulrich, R., Pedersen, A., Schmidt, R., Butler, A., Fransen, A., Klinge, D., Thomsen, M., Fälthammar, C.-G., Lindqvist, P.-A., Christenson, S., Holtet, J., Lybekk, B., Stein, T., Tanskanen, P., Lappalainen, K., and Wygant, J.: The Electric Field and Wave Experiment for the Cluster mission, Space Sci. Rev., 79/1-2, 137-156, 1997.

Hudson, M. K. and Mozer, F. S.: Electrostatic shocks, double layers, and anomalous resistivity in the magnetosphere, Geophys. Res. Lett., 5, 131-134, 1978.

Hwang, K.-J., Lynch, K. A., Carlson, C. W., Bonell, J. W., and Peria, W. J.: Fast Auroral Snapshot observations of perpendicular DC electric field structures in downward current regions: Implications, J. Geophys. Res., 111, A09206, doi:10.1029/2005JA011472, 2006.

Jasperse, J. R.: Ion heating, electron acceleration, and the selfconsistent E-field in downward auroral current regions, Geophys. Res. Lett., 25, 3485-3488, 1998.

Jasperse, J. R. and Grossbard, N. J.: The Alfvén-Fälthammar formula for the parallel E-field and its analogue in downward auroral-current regions, IEEE T. Plasma Sci., 28, 1874-1886, 2000.

Johansson, T., Figueiredo, S., Karlsson, T., Marklund, G., Fazakerley, A., Buchert, S., Lindqvist, P.-A., and Nilsson, H.: Intense high-altitude auroral electric fields - temporal and spatial characteristics, Ann. Geophys., 22, 2485-2495, 2004,

http://www.ann-geophys.net/22/2485/2004/.

Johansson, T., Karlsson, T., Marklund, G., Figueiredo, S.,
Lindqvist, P.-A., and Buchert, S.: A statistical study of intense electric fields at 4-7 RE geocentric distance using Cluster, Ann. Geophys., 23, 2579-2588, 2005, http://www.ann-geophys.net/23/2579/2005/.

Johansson, T., Marklund, G., Karlsson, T., Liléo, S., Lindqvist, P.A., Marchaudon, A., Nilsson, H., and Fazakerley, A.: On the profile of intense high-altitude auroral electric fields at magnetospheric boundaries, Ann. Geophys., 24, 1713-1723, 2006, http://www.ann-geophys.net/24/1713/2006/.

Karlsson, T. and Marklund, G.: A statistically study of intense lowaltitude electric fields observed by Freja, Geophys. Res. Lett., 23, 1005-1008, 1996.

Karlsson, T. and Marklund, G.: Simulations of effects of smallscale auroral current closure in the return current region, Physics of Space Plasmas, 15, 401-406, 1998.

Keiling, A., Wygant, J. R., Cattell, C. A., Mozer, F. S., and Russell, C. T.: The Global Morphology of Wave Poynting Flux: Powering the Aurora, Science, 299, 383-386, 2003.

Kimball, J. and Hallinan, T.: A morphological study of black vortex streets, J. Geophys. Res., 103, 14 683-14695, 1998.

Knight, S.: Parallel electric field, Planet. Space Sci., 21, 741-750, 1973.

Knudsen, D. J., Donovan, E. F., Cogger, L. L., Jackel, B., and Shaw, W. D.: Width and structure of mesoscale optical auroral arcs, Geophys. Res. Lett., 28, 705-708, 2001.

Lyons, L., Evans, D., and Lundin, R.: An observed relation between magnetic field aligned electric fields and downward electron energy fluxes in the vicinity of auroral forms, J. Geophys. Res., 84, 457-461, 1979.

Maggs, J. E. and Davis, T. N.: Measurements of the thickness of auroral structures, Planet. Space Sci., 216, 205-209, 1968.

Marklund, G., Sandahl, I., and Opgenoorth, H.: A study of the dynamics of a discrete auroral arc, Planet. Space Sci., 30, 179-197, 1982.

Marklund, G., Karlsson, T., and Clemmons, J.: On low-altitude particle acceleration and intense electric fields and their relation to black aurora, J. Geophys. Res., 102, 17 509-17 522, 1997.

Marklund, G., Ivchenko, N., Karlsson, T., Fazakerley, A., Dunlop, M., Lindquist, P.-A., Buchert, S., Owen, C., Taylor, M., Vaivalds, A., Carter, P., André, M., and Balogh, A.: Temporal evolution of the electric field accelerating electrons away from the auroral ionosphere, Nature, 414, 724-727, 2001.

Marklund, G., Johansson, T., Liléo, S., and Karlsson, T.: Cluster observations of an auroral potential and associated field-aligned current reconfiguration during thinning of the plasma sheet boundary layer, J. Geophys. Res., 112, A01208, doi:10.1029/2006JA011804, 2007.

McFadden, J. P., Carlson, C. W., and Ergun, R. E.: Microstructures of the auroral acceleration region as observed by FAST, J. Geophys. Res., 104, 14 453-14480, 1999.

Mozer, F. S.: Electric field mapping in the ionosphere at the equatorial plane, Planet. Space Sci., 18, 259-263, 1970.

Newell, P. T., Lyons, K. M., and Meng, C.-I.: A large survey of electron acceleration events, J. Geophys. Res., 101, 2599-2614, 1996.

Peria, W., Carlson, C., Ergun, R., McFadden, J., Bonnell, J., Elphic, R., and Strangeway, R.: Characteristics of field-aligned currents near the auroral acceleration region: FAST observations, in Magnetospheric Current Systems, Geophys. Monogr. Ser., 118, 181- 
$189,2000$.

Peticolas, L. M., Hallinan, T. J., Stenbeck-Nielsen, H. C., Bonnell, J. W., and Carlson, C. W.: A study of black aurora from aircraftbased optical observations and plasma measurements on FAST, J. Geophys. Res., 107, 1217, doi:10.1029/2001JA900157, 2002.

Pilipenko, V., Fedorov, E., Engebretson, M., and Yumoto, K.: Energy budget of Alfven wave interactions with the auroral acceleration region, J. Geophys. Res., 109, A10204, doi:10.1029/2004JA010440, 2004.

Rème, H., Bosqued, J. M., Sauvaud, J. A., Cros, A., Dandouras, J., Aoustin, C., Bouyssou, J., Camus, T., Cuvilo, J., Martz, C., Médale, J. L., Perrier, H., Romefort, D., Rouzaud, J., D’Uston, C., Möbius, E., Crocker, K., Granoff, M., Kistler, L. M., Popecki, M., Hovestadt, D., Klecker, B., Paschmann, G., Scholer, M., Carlson, C. W., Curtis, D. W., Lin, R. P., Mcfadden, J. P., Formisano, V., Amata, E., Bavassano-Cattaneo, M. B., Baldetti, P., Belluci, G., Bruno, R., Chionchio, G., Lellis, A. D., Shelley, E. G., Ghielmetti, A. G., Lennartsson, W., Korth, A., Rosenbauer, H., Lundin, R., Olsen, S., Parks, G. K., Mccarthy, M., and Balsiger, H.: The Cluster ion spectrometry (CIS) experiment, Space Sci. Rev., 19/1-2, 303-350, 1997.

Song, Y. and Lysak, R. L.: The physics in the auroral dynamo regions and auroral particle acceleration, Phys. Chem. Earth (C), 26, 33-42, doi:10.1016/S1464-1917(00)00087-8, 2001.

Stasiewicz, K. and Potemra, T.: Multiscale current structures obseved by Freja, J. Geophys. Res., 103, 4315-4325, 1998.
Stenbaek-Nielsen, H. C., Hallinan, T. J., Osborne, D. L., Kimball, J., Chaston, C., McFadden, J., Delory, G., Temerin, M., and Carlson, C.: Aircraft observations conjugate to FAST: Auroral arc thickness, Geophys. Res. Lett., 25, 2073-2076, 1998.

Temerin, M. and Carlson, C.: Current-Voltage relations in the downward auroral current region, Geophys. Res. Lett., 25, 23652368, 1998.

Temerin, M., Cerny, K., Lotko, W., and Mozer, F.: Observations of double layers and solitary waves in the auroral plasma, Phys. Rev. Lett., 48, 1175-1179, 1982.

Trondsen, T. S. and Cogger, L.: High-resolution television observations of black aurora, J. Geophys. Res., 102, 363-378, 1997.

Vaivads, A., Andre, M., Buchert, S., Eriksson, A., Olsson, A., Wahlund, J.-E., Janhunen, P., Marklund, G., Kistler, L., Mouikis, C., Winningham, D., Fazakerley, A., and Newell, P.: Discrete auroral arc, electroststic shock and suprathermal electrons powered by dispersive, anomalously resistive field line resonance, Geophys. Res. Lett., 30, 1106, doi:10.1029/2002GL016006, 2003.

Weimer, D. R. and Gurnett, D. A.: Large-Amplitude Auroral electric Fields Measured With DE 1, J. Geophys. Res., 98, 13 557$13564,1993$.

Weimer, D. R., Goertz, C. K., Gurnett, D. A., Maynard, N. C., and Burch, J. L.: Auroral zone electric fields from DE 1 and DE 2 at magnetic conjunctions, J. Geophys. Res., 90, 7479-7494, 1985. 